\title{
P-cadherin potentiates ligand-dependent EGFR and IGF-1R signaling in dysplastic and malignant oral keratinocytes
}

\author{
DESSEREE LYSNE, JAMES JOHNS, ANDREW WALKER, \\ RACHEL ECKER, CHRISTOPHER FOWLER and KATHRYN R. LAWSON \\ Department of Biochemistry, Midwestern University, Glendale, AZ 85308, USA
}

Received June 30, 2014; Accepted September 19, 2014

DOI: $10.3892 /$ or.2014.3545

\begin{abstract}
Oral and oropharyngeal cancer together constitute the sixth most common cancer worldwide, with over 400,000 new cases diagnosed each year. Early detection is paramount, as the 5-year survival rate for these cancers decreases markedly once tumors have become regionally invasive. In many tissues, including oral epithelia, neoplastic progression is accompanied by alterations in expression of the epithelial cell adhesion molecules E-cadherin and P-cadherin. Oral epithelia is one of only a few tissues in which P-cadherin levels have been noted to increase in dysplasia and well-differentiated carcinomas and decrease in advanced malignancies. In the present study, P-cadherin was overexpressed in both dysplastic and malignant oral keratinocytes to characterize the mechanisms by which aberrantly expressed P-cadherin may modulate tumor progression. We found that P-cadherin was able to potentiate ligand-dependent signaling of insulin-like growth factor 1 receptor (IGF-1R) in malignant keratinocytes and epidermal growth factor receptor (EGFR) in dysplastic cells. P-cadherin prolonged activation of the mitogen-activated protein kinase (MAPK) in both cell lines and also increased the magnitude of AKT phosphorylation in dysplastic cells. P-cadherin overexpression alone was sufficient to increase steady-state levels of the mesenchymal transcription factor Snail, increase cell motility and also induce morphological changes in dysplastic keratinocytes. Taken together, these data suggest that the aberrantly elevated levels of P-cadherin which occur in early oral tumor development may play a critical role in the augmentation of neoplastic signaling networks and in the further acquisition of aggressive phenotypes.
\end{abstract}

Correspondence to: Dr Kathryn R. Lawson, Department of Biochemistry, Midwestern University, 19555 North 59th Avenue, Glendale, AZ 85308, USA

E-mail: klawso@midwestern.edu

Key words: P-cadherin, oral squamous cell carcinoma, insulin-like growth factor 1 receptor, epidermal growth factor receptor, Snail, mitogen-activated protein kinase, AKT

\section{Introduction}

Oral and oropharyngeal cancer together constitute the sixth most common cancer worldwide, with $\sim 400,000$ new cases diagnosed each year (1). In spite of advances in screening and detection, the 5-year survival rate of these cancers, which stood at $53 \%$ in 1975 , has increased only marginally over the last forty years. The critical need to identify the mechanisms associated with early tumor development are underscored by the generally favorable patient outcomes associated with localized tumors and the precipitous decline in survival rates associated with regional and distant metastases (2).

In the course of tumor development, normal epithelial cells undergo a dedifferentiation program that results in alterations in signaling, gene expression and phenotype, a phenomenon that is collectively termed epithelial-to-mesenchymal transition (EMT). During the course of this transition, epithelial cells lose polarity, exhibit a fibroblastic morphology and begin expressing mesenchymal genes. These cells may also exhibit increased motility and invasive capabilities through increased expression of proteolytic enzymes. EMT also involves alterations in expression of a group of transmembrane calciumdependent adhesive glycoproteins called cadherins. E-cadherin is the best-studied member of the classical cadherin family and the prototypical cadherin of normal epithelia. E-cadherin maintains cellular architecture within the epithelia by linking actin cytoskeletons of adjacent cells. E-cadherin is also classified as a tumor suppressor, as expression of E-cadherin is lost relatively early in tumorigenesis $(3,4)$. The overexpression of E-cadherin in cell lines has been shown to restrain cell migration and metastatic signaling (4).

E-cadherin co-resides in the basal and suprabasal layers of oral epithelia with P-cadherin (5), a classical cadherin that has received far less study and whose role in epithelial tumor progression is far more nebulous. Unlike E-cadherin, which is unilaterally lost during epithelial tumor progression, $\mathrm{P}$-cadherin expression is elevated in certain advanced malignancies, such as those of breast and colon, and overexpression of P-cadherin in such cell lines promotes aggressiveness. In other cancers, such as bladder, it is the loss of P-cadherin that promotes tumor development (6-8).

The majority of studies regarding the role of P-cadherin in oral tumor progression are histological in nature and reveal a rare trend in the expression pattern of P-cadherin during the 
course of oral tumor development. Immunohistochemical analyses of human tissues and carcinogen-induced rodent tumors have demonstrated an abnormal increase in membrane-resident P-cadherin protein during oral dysplasia $(9,10)$ and persistent expression of membranous P-cadherin in well-differentiated oral tumors $(11,12)$ In more advanced oral malignancies, however, P-cadherin expression was decreased or absent $(11,12)$. In the present study, we explored the hypothesis that the transient increase in P-cadherin during early oral tumor development is not merely coincidental, but plays an active role in oral tumor progression.

How then may elevated levels of an endogenous epithelial cadherin facilitate aggressive cellular behavior? We addressed this question by investigating the ability of P-cadherin to modulate ligand-dependent signaling of two growth factor receptor tyrosine kinases: insulin-like growth factor 1 receptor (IGF-1R) and epidermal growth factor receptor (EGFR). Precedence for such a mechanism has been demonstrated for both E-cadherin, which modulates ligand-dependent signaling of IGF-1R, EGFR and fibroblast growth factor receptor (FGFR) (13-15) and $\mathrm{N}$-cadherin, which potentiates ligand-dependent FGFR signaling (16). Most recently, it has been demonstrated in ovarian cancer cells that P-cadherin and IGF-1R are able to form a functional complex upon stimulation of the gonadotropin-releasing hormone receptor (17).

In the present study, P-cadherin was overexpressed in both dysplastic and malignant oral cell lines, which were analyzed for growth factor signaling responses and phenotypic alterations. The results of this study provide the first evidence that P-cadherin can potentiate ligand-dependent signaling of both IGF-1R and EGFR. P-cadherin also modulated mesenchymal signaling, motility and in the case of dysplastic cells, morphology. These findings, together with existing histological data, suggest that transient increases in P-cadherin levels may be a means by which dysplastic and early neoplastic oral epithelial cells acquire additional aggressive phenotypes.

\section{Materials and methods}

Cell culture. The oral squamous carcinoma cell line UM-SCC22A (SCC22A) (obtained from Dr Thomas Carey, University of Michigan) was maintained in Minimum Essential Medium (Hyclone) supplemented with $10 \%$ fetal bovine serum (PAA Scientific) and $1 \%$ non-essential amino acids (Fisher). Dysplastic oral keratinocytes (DOK) (obtained from the European Collection of Cell Cultures, via Sigma-Aldrich) were maintained in Dulbecco's modified Eagle's medium (DMEM; PAA Scientific), supplemented with $10 \%$ fetal bovine serum and $5 \mu \mathrm{g} / \mathrm{ml}$ hydrocortisone (Sigma-Aldrich). All cells were maintained at $37^{\circ} \mathrm{C}$ and $5 \%$ $\mathrm{CO}_{2}$. For retroviral transduction, a cDNA encoding full-length human P-cadherin (18) was subcloned into the $S g f \mathrm{I}$ and $S f \mathrm{I}$ sites of the retroviral expression vector LZRS-MS-Neo (19). Production of amphotropic retrovirus and subsequent infection of SCC22A and DOK cells with the LZRS-Ms-Neo (empty control vector) or LZRS-Ms-Neo/P-cadherin constructs were performed as previously described (20). All transduced cells were selected and maintained in $400 \mu \mathrm{g} / \mathrm{ml} \mathrm{G} 418$ (Santa Cruz Biotechnology). Bright field photography of subconfluent SCC22A and DOK cell cultures, and of wounded cell monolayers, was performed utilizing an Axiovert 40 inverted microscope (Zeiss). Immunofluorescence photography was performed utilizing an Axio Imager Z1 fluorescence microscope with ApoTome attachment (Zeiss).

Western blotting. For both steady-state and time-course experiments, cells were grown to equal confluency and harvested in RIPA lysis buffer (150 mM sodium chloride, 1.0\% NP-40, $0.5 \%$ sodium deoxycholate, $0.1 \%$ sodium dodecyl sulfate and $50 \mathrm{mM}$ Tris, pH 8.0 TNE) supplemented with HALT phosphatase and protease inhibitor cocktail (Thermo Fisher Scientific). For signaling analyses of insulin-like growth factor (IGF) and epidermal growth factor (EGF), cells were serum starved $24 \mathrm{~h}$ prior to administration of growth factor. Cells were incubated with $10 \mathrm{ng} / \mathrm{ml} \mathrm{IGF-1} \mathrm{(Peprotech)} \mathrm{or} 50 \mathrm{ng} / \mathrm{ml}$ EGF (Peprotech) in warmed serum-free media at the time-points indicated. All incubations were performed at $37^{\circ} \mathrm{C}$ in a humidified $5 \% \mathrm{CO}_{2}$ incubator prior to cell lysis.

Lysates were quantitated using a BCA (Pierce) or DC (BioRad) protein assay. Equal quantities of protein were analyzed by SDS-PAGE and subjected to western immunoblotting. The bands of interest were identified utilizing the indicated primary antibody and HRP-conjugated goat anti-mouse or goat anti-rabbit secondary antibody (Jackson Laboratories) and the Pierce SuperSignal Chemiluminescent Reagent. Primary antibodies used in this study included goat anti-mouse antibodies directed against $\mathrm{P}$-cadherin (BD Transduction Laboratories), E-cadherin and $\beta$-catenin (Zymed), $\beta$-tubulin (Developmental Studies Hybridoma Bank, University of Iowa) and glyceraldehyde-3-phosphate dehydrogenase (GAPDH; Sigma-Aldrich). All goat anti-rabbit antibodies were purchased from Cell Signaling Technology and included antibodies against IGF-1R, EGFR, Snail, phospho-p42/p44 mitogen-activated protein kinase (MAPK), phospho-AKT and phospho-glycogen synthase kinase-3 $\beta$ (phospho-GSK-3 $\beta$ ). Blots were also visualized and quantitated on the Odyssey Imaging System (LI-COR) using DyeLight 680 and DyeLight 800 anti-mouse secondary antibodies. For film analysis, relative band intensities were quantitated using Image J software (http://rsb.info.nih.gov/ij/index.html) in accordance with software protocols.

Immunofluorescence staining. For immunofluorescence staining, SCC22A cells were grown for $48 \mathrm{~h}$ on glass coverslips and were switched to serum-free media $18 \mathrm{~h}$ prior to growth factor treatment. Cells were fixed immediately or treated with serum-free media containing $10 \mathrm{ng} / \mathrm{ml} \mathrm{IGF-1}$ for $2 \mathrm{~h}$ prior to fixation. Cells were fixed using $10 \%$ neutralbuffered formalin for $30 \mathrm{~min}$ and permeabilized with $5 \%$ Triton X-100 for $15 \mathrm{~min}$. Coverslips were blocked in $10 \%$ goat serum. Immunofluorescence was performed on cells using a mouse monoclonal antibody against $\mathrm{P}$-cadherin (BD Biosciences) and an Alexa 488 anti-mouse secondary (Invitrogen) or an anti-IGF-1R rabbit polyclonal antibody and an Alexa 594 anti-rabbit secondary antibody (Invitrogen). All slips were mounted in DAPI-containing mounting media (Vector Labs) and imaged using an ApoTome fluorescence microscope (Zeiss). Axiovision 4.8 software (Zeiss) was used to collect fluorescent images and to create merged images for each image set. 
A

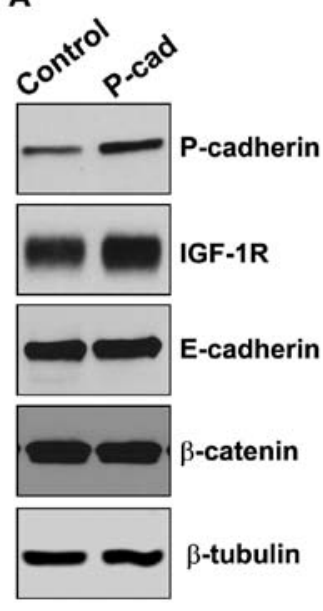

B

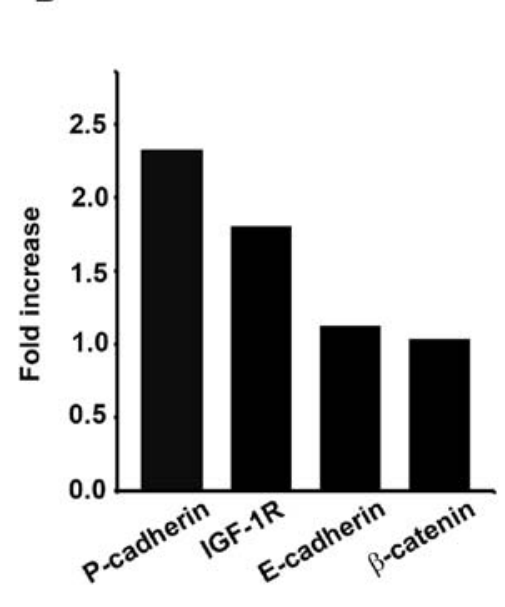

C

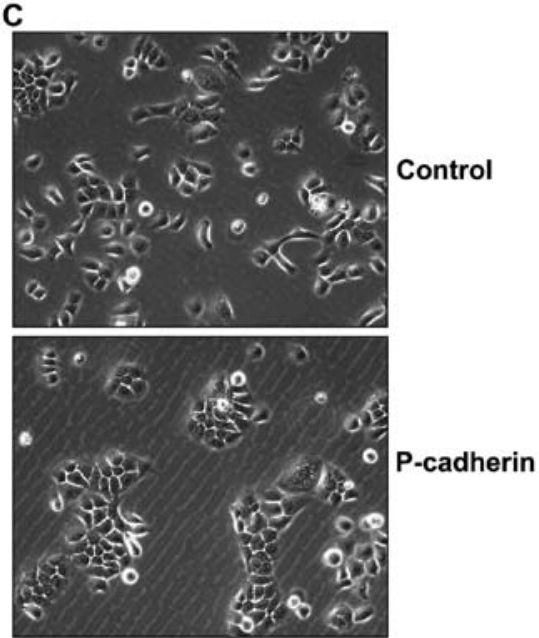

Figure 1. Expression of P-cadherin in retrovirally transduced cells. (A) Western blot analysis of adherens junction components (P-cadherin, E-cadherin and $\beta$-catenin) and insulin-like growth factor 1 receptor (IGF-1R). Oral squamous carcinoma SCC22A cells were retrovirally transduced with an empty expression vector (Control) or a vector directing overexpression of P-cadherin (P-cad). (B) Quantitation of protein levels in P-cadherin-expressing cells, normalized to respective levels found in the control cells. (C) Morphological analysis of SCC22A cells expressing endogenous (control) and elevated (P-cadherin) levels of P-cadherin. Cells were photographed $24 \mathrm{~h}$ after plating using a $10 \mathrm{X}$ objective.

Motility. For the wound healing assays, SCC22A cells were grown to confluence on grid-etched 60 -mm dishes (Fisher), and a single scratch was created using a pipette tip. Cells were photographed at the same location of the scratch over the course of $24 \mathrm{~h}$. The area of pixel closure was measured for each cell type by subtracting the initial scratch area from the final closing area $(24 \mathrm{~h})$. Values reported were normalized to the control and represent three independent experiments consisting of five plates for each cell type/time-point studied.

EMT array analysis. The Human EMT RT ${ }^{2}$ Profiler PCR Array (Qiagen, Redwood City, CA, USA) was utilized to examine the expression profile of 84 genes that are known to either regulate or affect processes related to epithelialto-mesenchymal transition. SCC22A cells transduced with control vector or P-cadherin cDNA were plated at equal densities and serum-starved for $24 \mathrm{~h}$ prior to RNA collection using the High-pure RNA Isolation kit (Roche, Indianapolis, IN, USA). RNA was isolated according to the manufacturer's instructions and quantitated using a Nanodrop spectrophotometer (Thermo Scientific). cDNA was prepared with the RT ${ }^{2}$ First Strand cDNA kit (Qiagen), utilizing $1 \mu \mathrm{g}$ RNA per reaction. Samples were run on an Applied Biosystems StepOne Plus qPCR (Invitrogen) using the $\mathrm{RT}^{2}$ SYBR-Green/ROX qPCR master mix (Qiagen) utilizing a final cDNA concentration of $0.5 \mathrm{ng} / \mu \mathrm{l}$ (5 $\mathrm{ng}$ per reaction). Data were normalized to the average $\mathrm{Ct}$ value of five housekeeping genes. Data analysis was performed utilizing the $2^{-\Delta \Delta \mathrm{Ct}}$ method by means of the $\mathrm{RT}^{2}$ Profiler PCR Data Analysis Template v4.0, available from the manufacturer's website (Qiagen).

\section{Results}

Transduction of oral squamous carcinoma cells with $P$-cadherin. IGF-1R has been shown to be moderately overexpressed in dysplastic oral lesions and highly expressed in malignant tumors (21). A recent studies by Cheung et al (17) demonstrated in an ovarian cancer cell model that gonadotropin-releasing hormone stimulates a functional association between P-cadherin and IGF-1R. To explore the possibility that P-cadherin may potentiate IGF-1R-related signaling in oral epithelia, we overexpressed P-cadherin in SCC22A oral squamous carcinoma cells. Cells were analyzed for E-cadherin and P-cadherin expression as well as the expression of IGF-1R and the P-cadherin-associated protein $\beta$-catenin (Fig. 1A and B). Cells transduced to overexpress P-cadherin displayed a 2-fold increase in $\mathrm{P}$-cadherin expression, and also displayed a near 2-fold increase in steady-state levels of IGF-1R protein. Other resident proteins of the adherens junction, E-cadherin and $\beta$-catenin, were unaltered by $\mathrm{P}$-cadherin overexpression. SCC22A cells exhibited an epithelial-like cellular morphology that was unaltered by expression of P-cadherin. P-cadherin expression did noticeably reduce cell scattering and favored greater colony formation (Fig. 1C).

$P$-cadherin potentiates IGF-1R-stimulated MAPK activation. To determine the effect of P-cadherin overexpression on IGF-1R-mediated signaling, we treated serum-deprived control and P-cadherin-overexpressing SCC22A cell line with IGF-1 for increasing time periods and analyzed the activating phosphorylation of MAPK and AKT (Fig. 2). P-cadherin increased both basal levels of MAPK phosphorylation (1.5 fold, Fig. 2B) and the magnitude of ligand-induced MAPK phosphorylation (9-fold compared to control), and also delayed return to steady-state levels through all time-points examined (Fig. 2B). Although ligand-dependent phosphorylation of AKT occurred in all cell lines examined, no P-cadherin-dependent alterations in the magnitude of phosphorylation were observed (Fig. 2A).

Independent internalization of IGF-1R and P-cadherin in response to IGF-1 signaling. E-cadherin has been show to alternatively co-internalize with ligand-stimulated growth-factor receptors (22) or remain at the cell membrane 

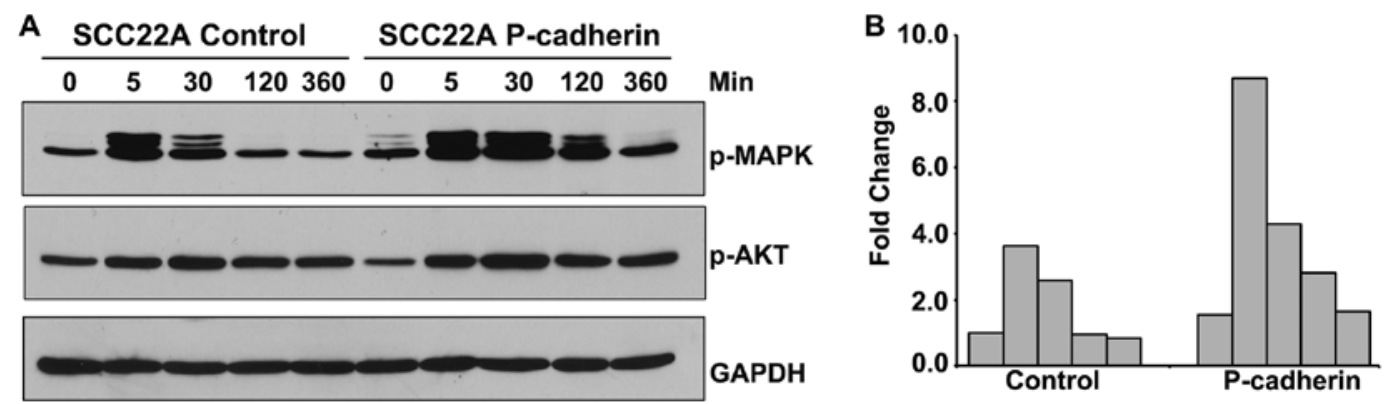

Figure 2. Induction of mitogen-activated protein kinase (MAPK) and AKT kinase activities by IGF-1. (A) Western blot analysis of MAPK and AKT phosphorylation in IGF-1-treated cells. Serum-starved control and P-cadherin-overexpressing oral squamous carcinoma SCC22A cells were treated with 10 ng/ ml IGF-1 and harvested at the indicated time-points. Lysates were prepared at indicated time-points and analyzed by immunoblotting for phospho-p42/p44 MAPK (ERK 1/2) or phospho-AKT. GAPDH was utilized as a loading control. (B) Relative quantitation of MAPK phosphorylation at 0,5,30,120 and 360 min (columns from left to right), as determined by densitometry. For both Control and P-cadherin-expressing cells, the signal was normalized to both GAPDH and time 0 values from the untreated control cells.

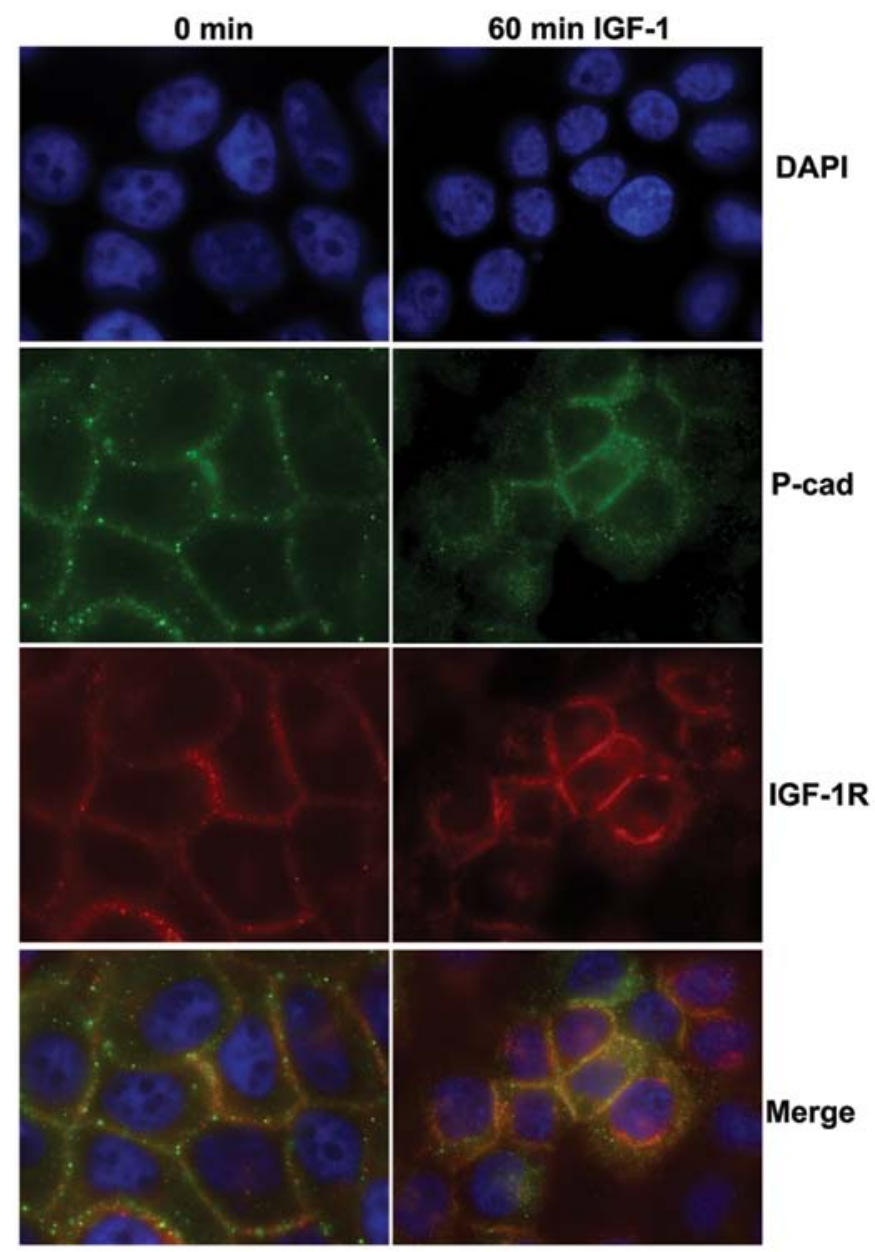

Figure 3. Internalization of P-cadherin (P-cad) and insulin-like growth factor 1 receptor (IGF-1R) upon IGF-1 administration. Oral squamous carcinoma SCC22A cells were grown for $48 \mathrm{~h}$ on glass coverslips and were switched to serum-free media $18 \mathrm{~h}$ prior to IGF-1 treatment $(10 \mathrm{ng} / \mathrm{ml})$. Untreated cells $(0 \mathrm{~min})$ were fixed at the time of IGF-1 administration to a separate culture or $1 \mathrm{~h}$ after treatment (60 min IGF-1). After sequential incubation with P-cadherin and IGF-1R antibodies (and their respective secondary antibodies), cells were mounted in DAPI-containing mounting media. AxioVision software was used to impart color to each image (blue, DAPI; green, P-cadherin; red, IGF-1R) and to composite images (Merge).

during growth factor receptor endocytosis (23). To identify the dynamics of P-cadherin and IGF-1R interactions and trafficking during IGF-1 stimulation, we examined the localization of each protein by immunofluorescence in the control and IGF-1-stimulated SCC22A cells (Fig. 3). In the untreated cells, both P-cadherin (green) and IGF-1R (red) were detected at points of cell-cell contact; however co-localization as determined by synergistic changes in fluorescence intensity was not observed. In the IGF-1-treated cells, both P-cadherin and IGF-1R were internalized. Trafficking of these molecules appeared to be somewhat independent, as P-cadherin staining was found throughout the cytoplasm, whereas IGF-1R staining was strong at both cell borders and around the nucleus.

P-cadherin expression increases transcription of EMT-related transcriptional regulators. The transient increase in P-cadherin expression in early tumor development suggests that P-cadherin may play an active role in facilitating epithelial-tomesenchymal transition. To identify EMT-related genes that were modulated by increased P-cadherin expression, an RNA profile array was used to examine the differential transcript levels of 84 different EMT-related genes. The panel screened for both effectors and regulators of EMT, and included genes involved in adhesion, migration, motility, as well as EMT-related transcriptional regulators. Since the majority of genes in the panel yielded no variance between the control and P-cadherin-expressing cells, we report only a relevant subset in Table I. In agreement with both the western blot analysis and SCC22A cell morphology, cadherin and catenin genes (E-cadherin, $\mathrm{N}$-cadherin, $\beta$-catenin) showed little change upon P-cadherin expression (Table I). An increase in gene expression was detected for all three members of the Snail family of EMT-associated transcriptional regulatory proteins and the EMT-associated transcriptional repressor Zeb1 (Table I). Transcript levels of vimentin and MMP-9, generally associated with mesenchymal phenotypes, were unaltered.

$P$-cadherin increases Snail protein levels independent of $I G F-1 R$ signaling. The qRT-PCR array analysis suggested that P-cadherin increased expression of the mesenchymal transcription factor Snail, which is also a known downstream target of IGF-1R- signaling (24). Levels of Snail protein were examined in serum-deprived SCC22A cells treated with IGF-1 for up to $6 \mathrm{~h}$. In the untreated control cells, Snail levels were 
Table I. Differential expression of EMT-related genes in the control and P-cadherin-overexpressing SCC22A cells.

\begin{tabular}{llc}
\hline Gene & \multicolumn{1}{c}{ Protein } & Fold change \\
\hline CDH1 & E-cadherin & 0.94 \\
CDH2 & N-cadherin & 1.13 \\
CTTNNB1 & $\beta$-catenin & 1.04 \\
MMP-9 & Matrix metalloproteinase-9 & 0.99 \\
SNAI1 & Snail homolog 1 (Drosophila) & 1.59 \\
SNAI2 & Snail homolog 2 (Drosophila) & 1.54 \\
SNAI3 & Snail homolog 3 (Drosophila) & 1.41 \\
TWIST1 & Twist homolog 1 (Drosophila) & 1.03 \\
VIM & Vimentin & 1.05 \\
ZEB1 & Zinc finger E-box binding homeobox 1 & 1.59
\end{tabular}

EMT, epithelial-to-mesenchymal transition.

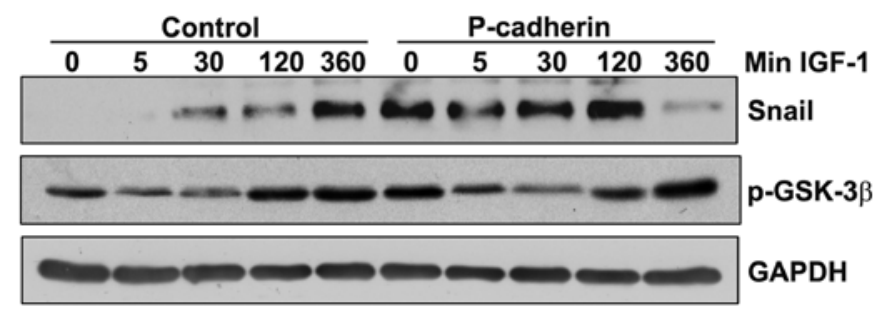

Figure 4. Western blot analysis of Snail and phosphorylated GSK-3 $\beta$ in IGF-1-treated oral squamous carcinoma SCC22A cells. Serum-starved control and P-cadherin-overexpressing cells were treated with $10 \mathrm{ng} / \mathrm{ml} \mathrm{IGF-1}$ and harvested at the indicated time-points. Lysates were prepared at the indicated time-points and analyzed by immunoblotting for Snail and serine-9 phosphorylated GSK-3 $\beta$. GAPDH was utilized as a loading control.

undetectable, but increased significantly by $6 \mathrm{~h}$ after IGF-1 administration (Fig. 4). P-cadherin overexpression alone was sufficient to increase basal levels of Snail (untreated P-cadherin cells) to levels comparable to the $6 \mathrm{~h}$ IGF-1 time-point in the control cells. IGF-1 was unable to stimulate further increases in Snail protein in the P-cadherin-expressing cells.

The 25-minute half-life of Snail protein is attributed to the phosphorylation of Snail at critical residues by glycogen synthase kinase-3 $\beta$ (GSK-3 $\beta$ ), which results in ubiquitination of Snail and its subsequent proteosome-mediated degradation (25). Accumulation of Snail occurs in response to the Serine- 9 phosphorylation of GSK-3 $\beta$ by multiple kinases, which inactivates GSK-3 $\beta$ and prevents phosphorylationdependent ubiquitination of Snail (26). In the SCC22A cells, profiles of GSK-3 $\beta$ phosphorylation across time-points were consistent between the control and P-cadherin-expressing cells, suggesting that the phosphorylation of GSK-3 $\beta$ at Serine-9 is unlikely to be the mechanism by which Snail levels are increased in P-cadherin-expressing cells (Fig. 4).

$P$-cadherin expression increases cell motility. Snail has been previously shown to be critical for cell motility in the highly dedifferentiated PCI152 oral squamous cell line (27). We utilized a wound healing assay to measure the differences in
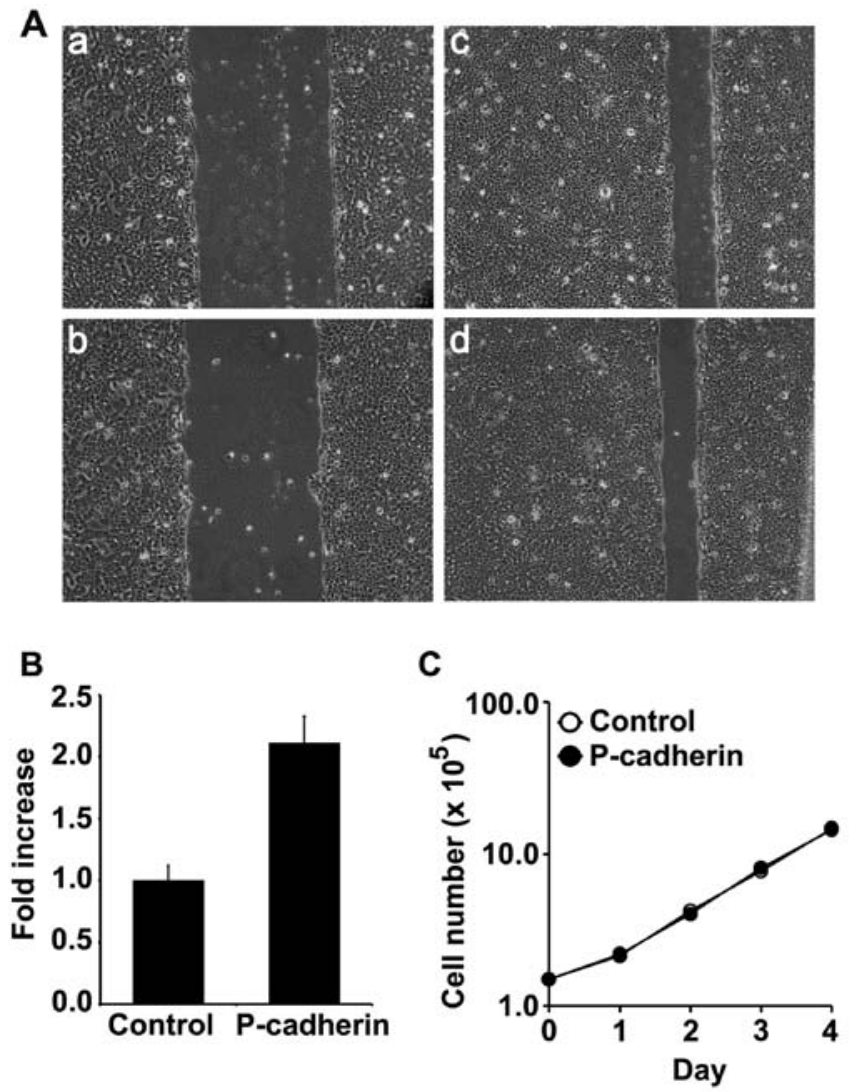

Figure 5. Effect of P-cadherin on motility and cell growth. (A) Wound healing analysis of control and P-cadherin-overexpressing oral squamous carcinoma SCC22A cells. Cells were plated to equal confluency and photographed at the same location 0 and $18 \mathrm{~h}$ after wounding with a pipette tip. Images are representative of control cells at 0 (panel a) and $18 \mathrm{~h}$ (panel b) or P-cadherinoverexpressing cells at 0 (panel c) and $18 \mathrm{~h}$ (panel d). (B) Relative motility of control and P-cadherin-expressing cells. Data are representative of five independently generated scratches for each time-point. (C) Growth analysis of control and P-cadherin-overexpressing cells. Cells were plated at $1.50 \times 10^{5}$ cells per chamber and counted on the days indicated. Data are representative of three independent hemocytometer counts from each of three independent cell platings.

motility between the control and the P-cadherin-expressing cells. Overexpression of P-cadherin conferred a 2-fold increase in motility compared to control cells (Fig. 5A). We did not observe P-cadherin-dependent alterations in motility as a result of IGF-1 administration (data not shown). Although P-cadherin increased cell motility, we found no evidence of $\mathrm{P}$-cadherin-dependent alterations in cell proliferation in the SCC22A cells as measured by growth curve analysis (Fig. 5C).

P-cadherin overexpression induces mesenchymal morphology in dysplastic oral keratinocytes. The increase observed in P-cadherin expression in dysplastic oral tissue (10) suggests the possibility that P-cadherin may play a stage-specific role in oral tumor progression. To better define the mechanisms by which P-cadherin may modulate signaling in dysplastic cells, we overexpressed P-cadherin in the DOK cell line. This cell line expresses E-cadherin and P-cadherin (Fig. 6A) but does not express the mesenchymal $\mathrm{N}$-cadherin in quantities detectable by western analysis (data not shown). Overexpression of $\mathrm{P}$-cadherin resulted in increased colony formation as observed in the SCC22A cells. In contrast to the SCC22A cells, however, 
A

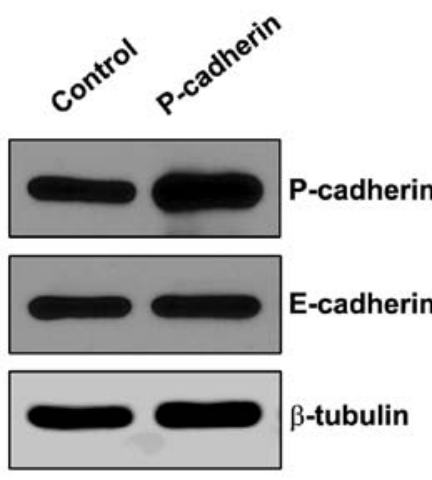

B

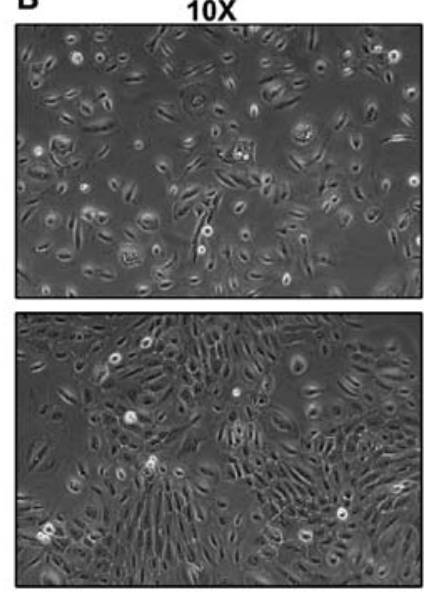

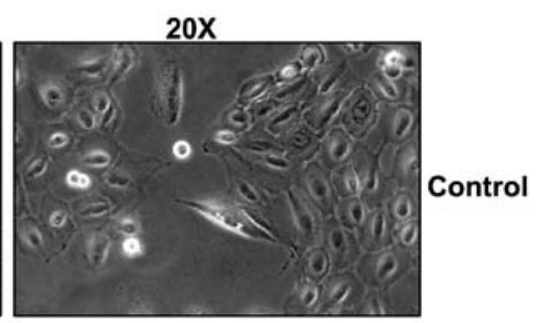

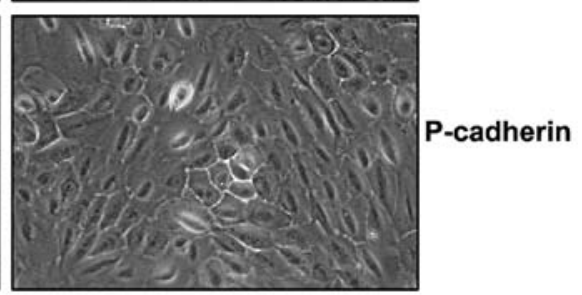

Figure 6. Western blot analysis and colony morphology of dysplastic oral keratinocytes (DOK). (A) Western blot analysis of P-cadherin and E-cadherin in DOK cells. $\beta$-tubulin was used as a loading control. (B) Morphology of control and P-cadherin-overexpressing DOK cells at x10 and x 20 magnification. Cells were plated in complete media and allowed to grow $48 \mathrm{~h}$ prior to imaging.
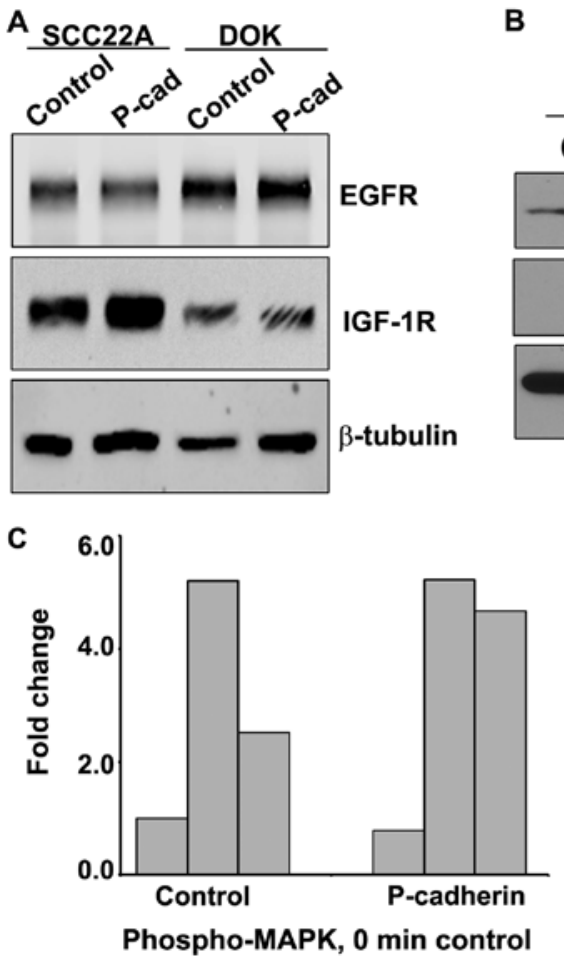

B
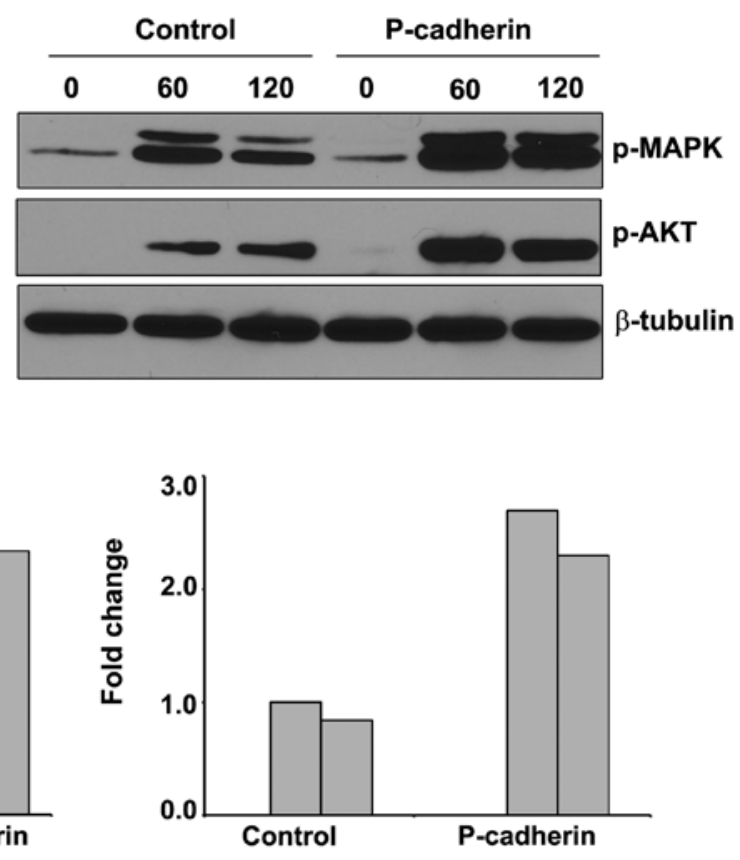

Phospho-AKT, 60 min control

Figure 7. Characterization of EGF-mediated signaling in dysplastic oral keratinocyte (DOK) cells. (A) Western blot analysis of epidermal growth factor receptor (EGFR) and insulin-like growth factor 1 receptor (IGF-1R) in oral squamous carcinoma SCC22A and DOK cells expressing normal or elevated levels of P-cadherin (P-cad). (B) Western blot analysis of mitogen-activated protein kinase (MAPK) and AKT signaling in DOK cells. Serum-starved control and P-cadherin-overexpressing DOK cells were treated with $50 \mathrm{ng} / \mu 1$ epidermal growth factor (EGF). Lysates were prepared at the indicated time-points and analyzed by immunoblotting for phospho-MAPK and phospho-AKT. $\beta$-tubulin was used as a loading control for both panels. (C) Relative quantitation of MAPK and AKT phosphorylation at 0,60 and $120 \mathrm{~min}$ (MAPK) or 60 and $120 \mathrm{~min}$ (AKT) (columns from left to right), as determined by densitometry. For MAPK analysis, all values were normalized to both $\beta$-tubulin and the 'Control 0 min' value of the MAPK signal. For AKT analysis, all values were normalized to both $\beta$-tubulin and the 'Control 60 min' value of the AKT signal (as densitometry at 0 min was not feasible).

P-cadherin overexpression in DOK cells had a profound effect on individual cell morphologies. DOK cells overexpressing P-cadherin exhibited a more elongated, fibroblastic phenotype compared to the control cell (Fig. 6B).

$P$-cadherin potentiates EGF-dependent MAPK and AKT signaling in dysplastic oral keratinocytes. In human oral epithelial tumors, increased expression and activity of EGFR is common, and appears to play a critical role in the acquisition of mesenchymal characteristics (28). A comparison of both EGFR and IGF-1R expression between DOK cells and the malignant carcinoma SCC22A cell line is shown in Fig. 7A. DOK cells exhibited much greater expression of EGFR and decreased expression of IGF-1R compared to the SCC22A cells. 
We examined the ability of P-cadherin to potentiate EGF-dependent signaling in DOK cells. Control or P-cadherinexpressing DOK cells were treated with EGF for 60 and 120 min and analyzed for both MAPK and AKT phosphorylation (Fig. 7B and C). Representative quantitation for each time-point analyzed is shown in Fig. 7C. P-cadherin did not appreciably amplify the magnitude of MAPK phosphorylation compared to control, but did prolong MAPK signaling at the 120-min time-point. P-cadherin expression did increase the magnitude of PI3 kinase signaling (as measured by AKT phosphorylation) by 3 -fold, compared to the 60 -min control value. Both control and P-cadherin DOK cells exhibited an $20 \%$ loss of signal between 60 and $120 \mathrm{~min}$, indicating that P-cadherin affected the initiation of EGFR signaling but did not play a protective role in the attenuation of AKT-mediated signaling.

\section{Discussion}

Oral cancer is one of the few tissues in which levels of membrane-resident P-cadherin is found to aberrantly increase in dysplasia and well-differentiated carcinomas and is subsequently lost in more advanced tumors $(10,29)$. In the present study, we demonstrated that aberrant expression of P-cadherin may in fact play a causative role during the early stages of tumor progression, by facilitating increases in motility and mesenchymal signaling and by strengthening IGF-1R or EGFR-initiated signal transduction cascades.

The increase in motility noted in P-cadherin-overexpressing SCC22A cells (Fig. 4) may be due to altered regulation of the P-cadherin binding partner p120 catenin and its subsequent modulation of Rho GTPases. These signaling molecules, which include the GTPases Rac, Rho and cdc42, can increase cell motility via the reorganization of cytoskeletal architecture and, in certain circumstances, also promote the dissolution of adherens junctions (30). In pancreatic cancer cell lines, P-cadherin overexpression altered p120 localization and, in a p120-dependent fashion, increased motility via the activation of both Rac and Cdc42 GTPases (31). In the present study, P-cadherin overexpression also increased expression of the Snail transcription factor, which has been shown by others to increase motility via the RhoA GTPase (32).

We were unable to determine the mechanism by which P-cadherin increased Snail protein levels. The activity of GSK-3 $\beta$, which phosphorylates and targets Snail for degradation, appeared to be unchanged between the control and P-cadherin cells (Fig. 4). One possible explanation is that P-cadherin indirectly stimulated the sequestration of active GSK-3 $\beta$ complexes into multivesicular endosomes, a phenomenon that has been shown to prolong the half-life of GSK-3 $\beta$ targets that would otherwise be targeted for degradation (33). Such regulation may have clinical implications, as Snail has been found at the invasive front of esophageal tumors and induces motility and invasion in esophageal squamous carcinoma cell lines (34).

We also demonstrated that overexpression of P-cadherin is able to increase the downstream signaling of two different ligand-activated growth factor receptors, IGF-1R and EGFR. In both dysplastic and malignant cells, P-cadherin expression prolonged MAPK activation in response to growth factor receptor signaling (Fig. 2B and C). In DOK cells, but not
SCC22A cells, P-cadherin overexpression greatly increased the magnitude of growth factor signaling through the PI3 kinase pathway (Fig. 7C). It is highly likely that the increased susceptibility of DOK cells to P-cadherin signaling is due to their dysplastic etiology, as they likely harbor comparatively fewer mutations and exhibit a decreased number of dysfunctional signaling pathways. The DOK cell line is an immortalized, nontumorigenic, moderately dysplastic oral keratinocyte cell line with normal Ha-ras, Ki-ras and $\mathrm{N}$-ras function (35). Unlike the SCC22A cells, growth-factor deprived DOK cells do not exhibit detectable levels of basal AKT phosphorylation (Figs. 2 and 7B). DOK cells, but not SCC22A cells, were also susceptible to morphological alterations as a result of P-cadherin overexpression (Fig. 1 and 6B). These data suggest that P-cadherin overexpression may have a more robust effect in oral precancers than it does in malignant neoplasms.

The ability of P-cadherin to potentiate IGF-1R and EGFR signaling may have profound consequences with respect to dysplastic epithelia. The temporal and spatial occurrence of aberrantly elevated P-cadherin expression is quite similar to the pattern of expression of EGFR in early oral tumor development. EGFR, which undergoes amplification in early oral dysplasia, is localized to the same basal layers of the epithelia where elevations in P-cadherin have been detected $(5,36)$. IGF-1R has also been found to increase during moderate to severe dysplasia (21). Unlike P-cadherin, increased expression of both EGFR and IGF-1R persists during tumor progression and thus, the increased downstream signaling responses conferred P-cadherin may occur only during dysplasia and early neoplasia. The MAPK and AKT signaling pathways mediate a number of tumor-associated activities, including growth, survival, cell motility and invasion (37-39). The increased activity of these pathways provided by the augmentation of growth factor signaling by P-cadherin may enhance tumor development at a critical stage and as such, may also provide new strategies for therapeutic intervention.

\section{Acknowledgements}

Support for this study was received from the Kenneth A. Suarez Summer Research Fellowship Program, Midwestern University College of Health Sciences and intramural funding from Midwestern University (Glendale, AZ, USA).

\section{References}

1. Warnakulasuriya S: Global epidemiology of oral and oropharyngeal cancer. Oral Oncol 45: 309-316, 2009.

2. Siegel R, Naishadham D and Jemal A: Cancer statistics, 2013. CA Cancer J Clin 63: 11-30, 2013.

3. Santos-García A, Abad-Hernández MM, Fonseca-Sánchez E, et al: E-caderin, laminin and collagen IV expression in the evolution from dysplasia to oral squamous cell carcinoma. Med Oral Path Oral Cir Bucal 11: E100-E105, 2006 (In English and Spanish)

4. Birchmeier W and Behrens J: Cadherin expression in carcinomas: role in the formation of cell junctions and the prevention of invasiveness. Biochim Biophys Acta 1198: 11-26, 1994.

5. Shimoyama Y, Hirohashi S, Hirano S, et al: Cadherin celladhesion molecules in human epithelial tissues and carcinomas. Cancer Res 49: 2128-2133, 1989.

6. Van Marck V, Stove C, Jacobs K, Van den Eynden G and Bracke M: P-cadherin in adhesion and invasion: Opposite roles in colon and bladder carcinoma. Int J Cancer 128: 1031-1044, 2011. 
7. Van Marck V, Stove C, Van Den Bossche K, et al: P-cadherin promotes cell-cell adhesion and counteracts invasion in human melanoma. Cancer Res 65: 8774-8783, 2005.

8. Paredes J, Albergaria A, Oliveira JT, Jerónimo C, Milanezi F and Schmitt FC: P-cadherin overexpression is an indicator of clinical outcome in invasive breast carcinomas and is associated with $\mathrm{CDH} 3$ promoter hypomethylation. Clin Cancer Res 11: 5869-5877, 2005.

9. Bagutti C, Speight PM and Watt FM: Comparison of integrin, cadherin and catenin expression in squamous cell carcinomas of the oral cavity. J Pathol 186: 8-16, 1998.

10. Williams HK, Sanders DS, Jankowski JA, Landini G and Brown AM: Expression of cadherins and catenins in oral epithelial dysplasia and squamous cell carcinoma. J Oral Pathol Med 27: 308-317, 1998

11. Muñoz-Guerra MF, Marazuela EG, Fernández-Contreras ME and Gamallo C: P-cadherin expression reduced in squamous cell carcinoma of the oral cavity: an indicatior of poor prognosis Cancer 103: 960-969, 2005

12. Lo Muzio L, Pannone G, Mignogna M, et al: P-cadherin expression predicts clinical outcome in oral squamous cell carcinomas. Histol Histopathol 19: 1089-1099, 2004.

13. Bedzhov I, Liszewska E, Kanzler B and Stemmler MP: IGF1R signaling is indispensable for preimplantation development and is activated via a novel function of E-cadherin. PLoS Genet 8 : e1002609, 2012.

14. Bryant DM, Wylie FG and Stow JL: Regulation of endocytosis, nuclear translocation and signaling of fibroblast growth factor receptor 1 by E-cadherin. Mol Biol Cell 16: 14-23, 2005.

15. Qian X, Karpova T, Sheppard AM, McNally J and Lowy DR E-cadherin-mediated adhesion inhibits ligand-dependent activation of diverse receptor tyrosine kinases. EMBO J 23 : $1739-1784,2004$

16. Suyama K, Shapiro I, Guttman M and Hazan RB: A signaling pathway leading to metastasis is controlled by N-cadherin and the FGF receptor. Cancer Cell 2: 301-314, 2002.

17. Cheung LW, Mak AS, Cheung AN, Ngan HY, Leung PC and Wong AS: P-cadherin cooperates with insulin-like growth factor-1 receptor to promote metastatic signaling of gonadotropin-releasing hormone in ovarian cancer via p120 catenin. Oncogene 30: 2964-2974, 2011.

18. Johnson KR, Lewis JE, Li D, et al: P- and E-cadherin are in separate complexes in cells expressing both cadherins. Exp Cell Res 207: 252-260, 1993

19. Ireton RC, Davis MA, Van Hengel J, et al: A novel role for p120 catenin in E-cadherin function. J Cell Biol 159: 465-476, 2002.

20. Maeda M, Johnson E, Mandal SH, et al: Expression of inappropriate cadherins by epithelial tumor cells promotes endocytosis and degradation of E-cadherin via competition for p120(ctn). Oncogene 25: 4595-4604, 2006

21. Joseph BK and Sundaram DB: Insulin-like growth factor-1 receptor expression in oral squamous cell carcinoma. J Clin Exp Invest 2, 354-361, 2011

22. Morali OG, Delmas V, Moore R, Jeanney C, Thiery JP and Larue L: IGF-II induces rapid beta-catenin relocation to the nucleus during epithelium to mesenchyme transition. Oncogene 20: 4942-4950, 2001.

23. Chavez MG, Buhr CA, Petrie WK, Wandinger-Ness A, Kusewitt DF and Hudson LG: Differential downregulation of E-cadherin and desmoglein by epidermal growth factor. Dermatol Res Pract 2012. 309587, 2012.
24. Kim HJ, Litzenburger BC, Cui X, et al: Constitutively active type I insulin-like growth factor receptor causes transformation and xenograft growth of immortalized mammary epithelial cells and is accompanied by an epithelial-to-mesenchymal transition mediated by NF- $\mathrm{B}$ and Snail. Mol Cell Biol 27: 3165-3175, 2007.

25. Zhou BP, Deng J, Xia W, et al: Dual regulation of Snail by GSK-3beta-mediated phosphorylation in control of epithelial-mesenchymal transition. Nat Cell Biol 6: 931-940, 2004.

26. Sutherland C, Leighton IA and Cohen P: Inactivation of glycogen synthase kinase-3 beta by phosphorylation: new kinase connections in insulin and growth-factor signalling. Biochem J 296: 15-19, 1993.

27. Bauer K, Dowejko A, Bosserhoff AK, Reichert T and Bauer RJ: P-cadherin induces an epithelial-like phenotype in oral squamous cell carcinoma by GSK-3beta-mediated Snail phosphorylation. Carcinogenesis 30: 1781-1788, 2009.

28. Hiraishi Y, Wada T, Nakatani K, Negoro K and Fujita S: Immunohistochemical expression of EGFR and p-EGFR in oral squamous cell carcinomas. Pathol Oncol Res 12: 87-91, 2006.

29. Lo Muzio L, Campisi G, Farina A, et al: P-cadherin expression and survival rate in oral squamous cell carcinoma: an immunohistochemical study. BMC Cancer 5: 63, 2005.

30. Lozano E, Betson M and Braga VM: Tumor progression: small GTPases and loss of cell-cell adhesion. Bioessays 25: 452-463, 2003.

31. Taniuchi K, Nakagawa H, Hosokawa M, et al: Overexpressed P-cadherin/CDH3 promotes motility of pancreatic cancer cells by interacting with p120ctn and activating rho-family GTPases. Cancer Res 65: 3092-3099, 2005.

32. Zhang A, Wang Q, Han Z, et al: Reduced expression of Snail decreases breast cancer cell motility by downregulating the expression and inhibiting the activity of RhoA GTPase. Oncol Lett 6: 339-346, 2013.

33. Taelman VF, Dobrowolski R, Plouhinec JL, et al: Wnt signaling requires sequestration of glycogen synthase kinase 3 inside multivesicular endosomes. Cell 143: 1136-1148, 2010.

34. Usami Y, Satake S, Nakayama F, et al: Snail-associated epithelial-mesenchymal transition promotes oesophageal squamous cell carcinoma motility and progression. J Pathol 215 : 330-339, 2008.

35. Chang SE, Foster S, Betts D and Marnock WE: DOK, a cell line established from human dysplastic oral mucosa, shows a partially transformed non-malignant phenotype. Int J Cancer 52: 896-902, 1992

36. Tsui IF, Poh CF, Garnis C, Rosin MP, Zhang L and Lam WL: Multiple pathways in the FGF signaling network are frequently deregulated by gene amplification in oral dysplasias. Int J Cancer 125: 2219-2228, 2009.

37. Grille SJ, Bellacosa A, Upson J, et al: The protein kinase Akt induces epithelial mesenchymal transition and promotes enhanced motility and invasiveness of squamous cell carcinoma lines. Cancer Res 63: 2172-2178, 2003.

38. Reddy KB, Krueger JS, Kondapaka SB and Diglio CA Mitogen-activated protein kinase (MAPK) regulates the expression of progelatinase B (MMP-9) in breast epithelial cells. Int J Cancer 82: 268-273, 1999.

39. Klemke RL, Cai S, Giannini AL, Gallagher PJ, De Lanerolle P and Cheresh DA: Regulation of cell motility by mitogen-activated protein kinase. J Cell Biol 137: 481-492, 1997. 